\title{
The charge distribution on a protein surface determines whether productive or futile encounter complexes are formed
}

Di Savino, A.; Foerster, J.M.; Ullmann, G.M.; Ubbink, M.

\section{Citation}

Di Savino, A., Foerster, J. M., Ullmann, G. M., \& Ubbink, M. (2021). The charge distribution on a protein surface determines whether productive or futile encounter complexes are formed.

Biochemistry, 60(10), 747-755. doi:10.1021/acs.biochem.1c00021

Version: $\quad$ Publisher's Version

License: $\quad$ Creative Commons CC BY-NC-ND 4.0 license

Downloaded from: https://hdl.handle.net/1887/3245479

Note: To cite this publication please use the final published version (if applicable). 


\title{
The Charge Distribution on a Protein Surface Determines Whether Productive or Futile Encounter Complexes Are Formed
}

\author{
Antonella Di Savino, Johannes M. Foerster, G. Matthias Ullmann, and Marcellus Ubbink*
}

Cite This: Biochemistry 2021, 60, 747-755

Read Online

ABSTRACT: Protein complex formation depends strongly on electrostatic interactions. The distribution of charges on the surface of redox proteins is often optimized by evolution to guide recognition and binding. To test the degree to which the electrostatic interactions between cytochrome $c$ peroxidase $(\mathrm{CcP})$ and cytochrome $c(\mathrm{Cc})$ are optimized, we produced five $\mathrm{CcP}$ variants, each with a different charge distribution on the surface. Monte Carlo simulations show that the addition of negative charges attracts Cc to the new patches, and the neutralization of the charges in the regular, stereospecific binding site for $\mathrm{Cc}$ abolishes the electrostatic interactions in that region entirely. For CcP variants with the charges in the regular binding site intact, additional negative patches slightly enhance productive complex formation, despite disrupting the optimized charge distribution. Removal of the charges in the regular binding site results in a dramatic decrease in the complex formation rate, even in the presence of highly negative patches elsewhere on the surface. We conclude that additional charge patches can result in either productive or futile encounter complexes, depending on whether negative residues are located also in the regular binding site.

$\mathrm{P}$ rotein complex formation proceeds through several steps. Freely diffusing proteins can collide and either move away again or form an encounter complex, which may result in the formation of the stereospecific and active complex. The encounter complex is an ensemble of configurations in which the proteins sample each other's surface. ${ }^{1}$ Formation is initially driven by electrostatic interactions, but as the proteins get close, hydrophobic interactions can also come into play. $^{2-9}$ If the encounter results in the stereospecific complex, it is called productive; otherwise, it is futile. ${ }^{10,11}$ The rate of formation of the active complex defines the association rate constant $\left(k_{\mathrm{a}}\right)$. If complex formation is not optimized, $k_{\mathrm{a}}$ can be $4-5$ orders of magnitude lower than the number of collisions, because in most cases the proteins do not collide with binding sides oriented toward each other. Highly optimized protein complexes have $k_{\mathrm{a}}$ values close to the diffusion limit, due to strong electrostatic preorientation. ${ }^{12}$ Harel et al. ${ }^{10}$ studied complex formation of TEM1- $\beta$-lactamase (TEM1) and its inhibitor, $\beta$-lactamase inhibitor protein (BLIP). They showed that charge mutations can enhance productive encounter complex formation, thus increasing the association rate without affecting the dissociation rate. However, other mutations modify the encounter complex without affecting the association rate or increase $k_{\mathrm{a}}$ without altering the encounter complex. The fractions of productive and futile encounter complexes can be altered with charge mutations on the proteins surface, even if they are far from the stereospecific binding site. By enhancing or disrupting a "pathway" from the encounter site to the binding site in the stereospecific complex, they can either facilitate the formation of the active complex or promote the early dissociation of the proteins. ${ }^{13}$ The relation between productive versus futile encounters and such electrostatic pathways on the protein surface was also suggested for the complex of cytochrome P450cam and putidaredoxin. ${ }^{14}$ Despite their name, futile encounter complexes can have a role in protein functionality. Recently, it was shown that futile encounter complexes can regulate the activity of a protein complex through the formation of competitive encounters with a third protein. 15

Electron transfer (ET) protein complexes often are highly optimized in complex formation and usually have a high fraction of the encounter state. We selected the complex formed by cytochrome $c$ (Cc) (CYC1, UniProtKB P00044) and cytochrome $c$ peroxidase $(\mathrm{CcP})$ (CCP1, UniProtKB P00431) to study the importance of the charge distribution on the protein surface in relation to the rate of formation of the stereospecific complex. The protein complex formed by $\mathrm{Cc}$ and $\mathrm{CcP}$ has been extensively studied and has become a paradigm for biological ET. CcP catalyzes the reduction of $\mathrm{H}_{2} \mathrm{O}_{2}$ in yeast mitochondria through a cycle of reactions. The crystallographic structure of the complex with Cc published in 1992 represents the stereospecific complex, ${ }^{16}$ and a second low-affinity binding

Received: January 7, 2021

Revised: February 22, 2021

Published: March 1, 2021 
site is present at ionic strength values below $\sim 100 \mathrm{mM}^{17-20}$ Electrostatic interactions are very important in the association reaction. ${ }^{18,20-23}$ Erman and co-workers ${ }^{24-26}$ measured the rate of association between $\mathrm{Cc}$ and several charge-reversal mutants of $\mathrm{CcP}$, showing that, interestingly, not only residues close to the stereospecific binding site but also more distant ones have a role in the association. In a study from our groups, the charge distribution on the surface of $\mathrm{CcP}$ was changed strongly by addition of a negative patch on one side of $\mathrm{CcP} .^{27}$ Monte Carlo simulations and paramagnetic relaxation enhancement (PRE) experiments showed that $\mathrm{Cc}$ interacts with the added patch, enlarging the surface visited in the encounter complex. The added charges also slightly enhance $k_{\mathrm{a}}$. To investigate further the degree to which the distribution of charges on the surface of $\mathrm{CcP}$ affects the association with $\mathrm{Cc}$, here we analyze four additional mutants of $\mathrm{CcP}$ with radically different charge distributions. Monte Carlo simulations show that the mutations alter the encounter complex, attracting $\mathrm{Cc}_{\mathrm{c}}$ to the added negative patches. The association rates were measured through stopped flow experiments for a wide range of ionic strength values. Surprisingly, the added patches turn out to yield either productive or futile encounters, depending on whether the stereospecific site has its charges intact.

\section{MATERIALS AND METHODS}

Mutagenesis. Saccharomyces cerevisiae CcP (CCP1, UniProtKB P00431) considered the wild type (WT) in this study ( $\mathrm{CcP}$ A) contains the $\mathrm{C} 128 \mathrm{~A}$ mutation to avoid dimerization and the additional sequence MKT at the N-terminus for enhanced production levels. ${ }^{28,29}$ The gene was subcloned into a pET28a(+) vector. $^{30} \mathrm{CcP}$ B additionally has the $\mathrm{K} 21 \mathrm{E}$, $\mathrm{K} 29 \mathrm{E}, \mathrm{K} 90 \mathrm{E}$, and K97E mutations. ${ }^{27} \mathrm{CcP} \mathrm{C}$ contains the same mutations as CcP_B and D35N, E36Q E119Q, E202Q, E210Q E215Q D225N, and E291Q that neutralize the charges of the WT binding site. CcP_D has mutations K75E, K76Q N79D, Q87E, K91Q K98Q and N142D in comparison to CcP_A. CcP_E has the same mutations as mutant CcP_D and the mutations that neutralize the charges of the WT $\bar{b}$ inding site as in $\mathrm{CcP}_{-} \mathrm{C}$. CcP_F has only the neutralization mutations for the WT binding site (see Table S1). The DNA constructs were produced by a commercial company and verified by DNA sequencing.

Protein Production. CcP was expressed in Escherichia coli BL21 Star (DE3)pLysS (Life Technologies Europe BV, Bleiswijk, The Netherlands). The production and purification were performed as previously described ${ }^{28,30,31}$ with some differences according to the $\mathrm{CcP}$ variant purified. Buffers with different ionic strength values were used to build the gradient for elution during anion-exchange chromatography: $50 \mathrm{mM}$ potassium phosphate (pH 5) and $500 \mathrm{mM}$ potassium phosphate ( $\mathrm{pH} 5)$ for CcP_A and CcP_C and CcP_E; 500 $\mathrm{mM} \mathrm{KCl}$ was added to the second buffer to elute $\mathrm{CcP}_{-} \mathrm{B}$ and CcP_D. The purification of CcP_F comprised a precipitation step with $40 \%(\mathrm{w} / \mathrm{w})$ ammonium sulfate at $25^{\circ} \mathrm{C}$, followed by dialysis against $20 \mathrm{mM}$ potassium phosphate ( $\mathrm{pH} 5$ ) overnight. Finally, the sample was loaded on an SP column; the column was washed with $20 \mathrm{mM}$ potassium phosphate $(\mathrm{pH} \mathrm{5)}$ and eluted with 20 column volumes of a gradient to $20 \mathrm{mM}$ potassium phosphate and $1 \mathrm{M} \mathrm{NaCl}(\mathrm{pH} \mathrm{5)}$. S. cerevisiae Cc (CYC1, UniProtKB P00044) was expressed using the iso-1cytochrome $c$ gene in a pUC19-based plasmid and purified following the protocols previously described. ${ }^{30,32}$
Monte Carlo Simulations. The structures were prepared, and the Monte Carlo docking simulations were performed as described in ref 27. The structures of $\mathrm{CcP}$ and $\mathrm{Cc}$ from Protein Data Bank (PDB) entry $2 \mathrm{PCC}^{16}$ were used as a basis for the simulations. The hydrogens of the proteins were added with HBUILD $^{33}$ in CHARMM $^{34}$ and minimized with the CHARMM force field, ${ }^{35}$ while the heavy atoms were fixed. The hemes within the protein were considered to be in the oxidized state. For each version of $\mathrm{CcP}\left(\mathrm{CcP}_{-} \mathrm{A}-\mathrm{CcP}_{-} \mathrm{F}\right)$, the mutations, described in Mutagenesis, were introduced using Pymol. $^{36}$ The electrostatic potential was calculated with $\mathrm{APBS}^{37}$ for each CcP mutant separately. The potential was calculated in the presence of $120 \mathrm{mM} \mathrm{NaCl}$ at a temperature of $303 \mathrm{~K}$. The Monte Carlo docking simulations were performed with the program suite MCMap. ${ }^{38}$ Separate simulations were performed for each $\mathrm{CcP}$ mutant considering its respective electrostatic potential. In the simulation, $\mathrm{Cc}$ (ligand) moves randomly in the electrostatic potential of $\mathrm{CcP}$ (receptor). From each simulation, 5000 randomly chosen encounters were used for further analysis. A Cc:CcP protein pair was considered as an encounter complex if the distance between at least two atoms of each protein was $<3 \AA$.

Kinetic Measurements. The results of the kinetics measured for the $\mathrm{Cc}: \mathrm{CcP} \mathrm{A}_{-}$and $\mathrm{Cc}: \mathrm{CcP} \_\mathrm{B}$ complexes at ionic strengths from 752 to $122 \mathrm{mM}$ were presented in our previous work. $^{27}$ The same protocols were followed to measure the reactions between $\mathrm{Cc}$ and the other mutants of $\mathrm{CcP}$. The kinetic measurements were performed at ionic strengths 752, $622,502,392,292,192,122$, and $44 \mathrm{mM}$ on a SX20 stopped flow spectrometer (AppliedPhotophysics, Leatherhead, Surrey, U.K.) with a $1.2 \mathrm{~ms}$ dead time at room temperature following the absorbance at $416 \mathrm{~nm}$, which indicates oxidation of Cc. ${ }^{39}$ Equimolar solutions of $\mathrm{Cc}$ and $\mathrm{CcP}$ were mixed to a final concentration of $0.5 \mu \mathrm{M}$ or $1.0 \mu \mathrm{M}$ of both $\mathrm{Cc}$ and $\mathrm{CcP}$.

Kinetic Data Analysis. The stopped flow data were fitted to eq 1 as previously described: ${ }^{27}$

$$
\Delta A_{416}=B_{0} \Delta \varepsilon_{416}\left(1-\frac{1}{1+k_{\mathrm{obs}} B_{0} t}\right)+C
$$

where $B_{0}$ is the starting concentration of $\mathrm{Cc}\left(\mathrm{Fe}^{2+}\right)$ and $\mathrm{CpdI}$ after mixing, $\Delta \varepsilon_{416}$ is the difference in extinction coefficient at $416 \mathrm{~nm}$ for oxidized and reduced $\mathrm{Cc},-40 \mathrm{mM}^{-1} \mathrm{~cm}^{-1}$, $k_{\text {obs }}$ (inverse seconds) is the observed rate constant of reduction, $t$ is the time (seconds), and $C$ corrects for the baseline voltage of the spectrometer.

An independent analysis of the stopped flow data was performed through simulations. Kinetic simulations using GNU Octave ${ }^{43}$ were performed to simulate the experimental measurement of the kinetics obtained from a stopped flow measurement. The script is included in the Supporting Information (Script S1). To do so, reactions 2-4 (see Results) that influence the absorbance at $416 \mathrm{~nm}$ during the catalytic cycle were considered. The following differential equations were used to describe the change in the concentration of each species in solution during the cycle:

$$
\begin{aligned}
\frac{\mathrm{d}\left[\mathrm{Cc}\left(\mathrm{Fe}^{2+}\right)\right]}{\mathrm{d} t}= & -k(\mathrm{II})\left[\mathrm{Cc}\left(\mathrm{Fe}^{2+}\right)\right][\mathrm{CpdI}] \\
& -k(\mathrm{III})\left[\mathrm{Cc}\left(\mathrm{Fe}^{2+}\right)\right][\mathrm{CpdII}]
\end{aligned}
$$




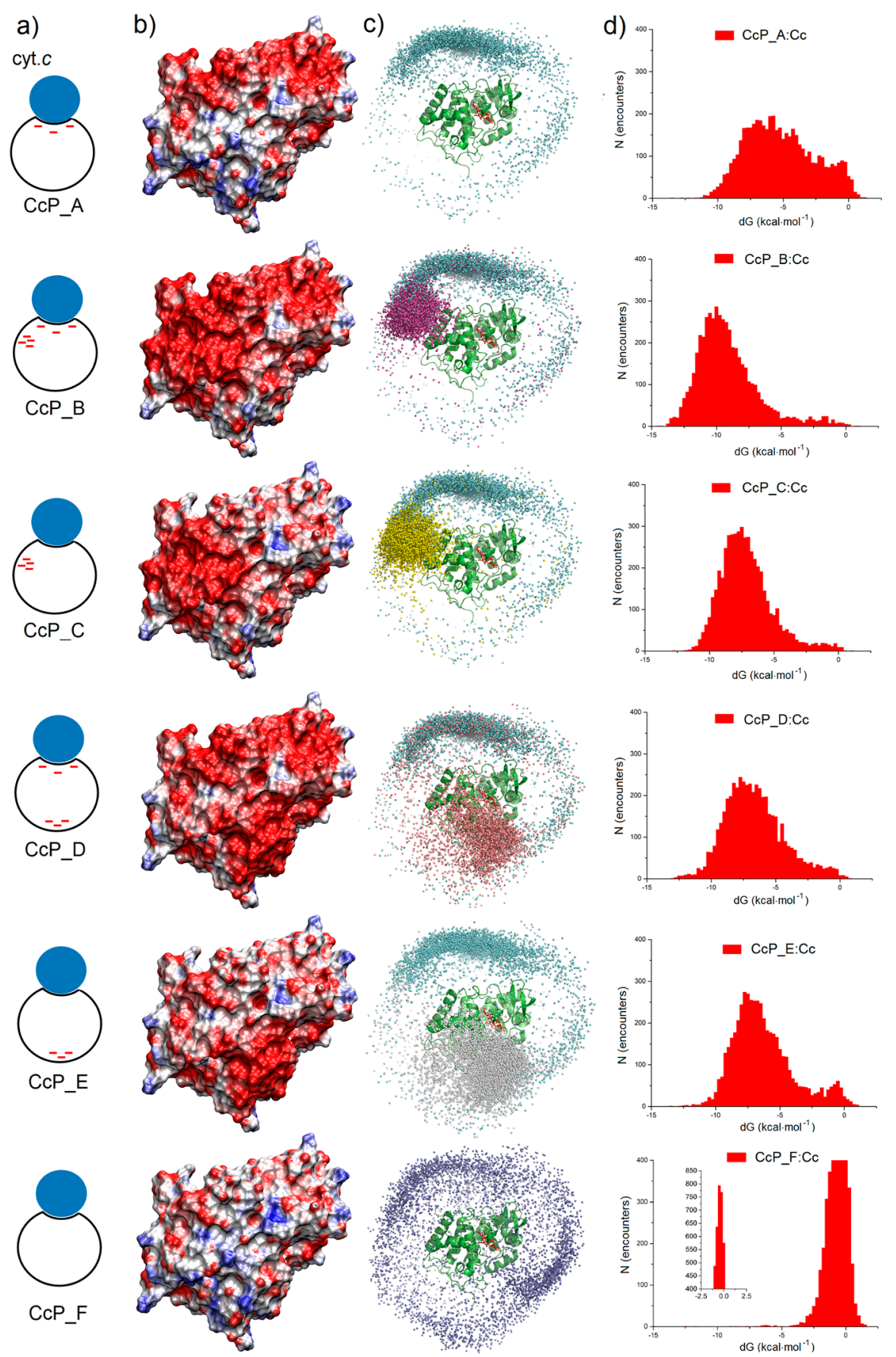

Figure 1. CcP variants. (a) Schematic representation of the complexes formed by $\mathrm{Cc}$ and the $\mathrm{CcP}$ variants. $\mathrm{Cc}$ is represented as a blue circle bound at the stereospecific binding site, and the $\mathrm{CcP}$ variants are represented as empty circles with the negative charges on the surface indicated as red dashes. (b) Electrostatic potential plotted on the surface of the CcP variants ranging from -5 (red) to $5 \mathrm{kcal} / \mathrm{e}^{\circ}$ (blue) at an ionic strength of 120 $\mathrm{mM}$. (c) Structure of CcP (green ribbon, red heme) surrounded by the centers of the mass of Cc in the ensemble of encounters of the Cc:CcP_A (cyan), Cc:CcP_B (magenta), Cc:CcP_C (yellow), Cc:CcP_D (salmon), Cc:CcP_E (gray), and Cc:CcP_F (purple) complexes as obtained from rigid body Monte Carlo simulations. ( $\overline{\mathrm{d}})$ Energy distribution of the encounter complexes between $\mathrm{Cc}$ and the $\mathrm{CcP}$ variants as obtained from rigid body Monte Carlo simulations. The inset shows the entire distribution for CcP_F using a different vertical scale.

$$
\begin{aligned}
& \frac{\mathrm{d}[\mathrm{CpdI}]}{\mathrm{d} t}=-k(\mathrm{II})\left[\mathrm{Cc}\left(\mathrm{Fe}^{2+}\right)\right][\mathrm{CpdI}]+k(\mathrm{IV})[\mathrm{CpdIII}]^{2} \quad \frac{\mathrm{d}[\mathrm{CpdII}]}{\mathrm{d} t}=k(\mathrm{II})\left[\mathrm{Cc}\left(\mathrm{Fe}^{2+}\right)\right][\mathrm{CpdI}]-k(\mathrm{III})\left[\mathrm{Cc}\left(\mathrm{Fe}^{2+}\right)\right][\mathrm{CpdII}] \\
& -k(\mathrm{IV})[\mathrm{CpdII}]^{2}
\end{aligned}
$$

$$
\frac{\mathrm{d}\left[\mathrm{Cc}\left(\mathrm{Fe}^{3+}\right)\right]}{\mathrm{d} t}=k(\mathrm{II})\left[\mathrm{Cc}\left(\mathrm{Fe}^{2+}\right)\right][\mathrm{CpdI}]+k(\mathrm{III})\left[\mathrm{Cc}\left(\mathrm{Fe}^{2+}\right)\right][\mathrm{CpdII}]
$$

$$
\frac{\mathrm{d}[\mathrm{CcP}]}{\mathrm{d} t}=k(\mathrm{IV})[\mathrm{CpdII}]^{2}+k(\mathrm{III})\left[\mathrm{Cc}\left(\mathrm{Fe}^{2+}\right)\right][\mathrm{CpdII}]
$$


From the simulated concentrations, the stopped flow trace is re-created by summing the contributions of each component to the absorbance at $416 \mathrm{~nm}$ following the Beer-Lambert law:

$$
\begin{aligned}
A_{416}= & \varepsilon_{\mathrm{Cc}\left(\mathrm{Fe}^{2+}\right)}\left[\mathrm{Cc}\left(\mathrm{Fe}^{2+}\right)\right]+\varepsilon_{\mathrm{CpdI}}[\mathrm{CpdI}] \\
& +\varepsilon_{\mathrm{Cc}\left(\mathrm{Fe}^{3+}\right)}\left[\mathrm{Cc}\left(\mathrm{Fe}^{3+}\right)\right]+\varepsilon_{\mathrm{CpdII}}[\mathrm{CpdII}]+\varepsilon_{\mathrm{CcP}}[\mathrm{CcP}] \\
& +C
\end{aligned}
$$

The extinction coefficients at $416 \mathrm{~nm}$ are $129.1 \mathrm{mM}^{-1} \mathrm{~cm}^{-1}$ for $\mathrm{Cc}\left(\mathrm{Fe}^{2+}\right),{ }^{44} 88.8 \mathrm{mM}^{-1} \mathrm{~cm}^{-1}$ for $\mathrm{Cc}\left(\mathrm{Fe}^{3+}\right),{ }^{44}$ and the values in Table S2 for CcP and CpdI. The extinction coefficient for CdpII was considered to be the same as for the corresponding CpdI. The baseline correction term $C$ was optimized manually (variable prod in the script in the Supporting Information). The values of $k(\mathrm{II}), k(\mathrm{III})$, and $k(\mathrm{IV})$ were independently changed until the simulation was a good fit as judged by visual inspection. The main error for the stopped flow measurement is caused by the error in the concentration of $\mathrm{Cc}$ and CpdI. Repeated measurement of the concentration of the samples resulted in a maximum deviation of $20 \%$ from the theoretical one. To take this into account in the simulations, every kinetic measurement, consisting of at least three replicates per salt concentration, was simulated at the expected experimental concentration, and at a concentration of $\mathrm{CcP}$ that is $20 \%$ lower. The rates of the reactions at a particular salt concentration were calculated as the average of the values obtained from the single simulations. The error of the rates at each salt concentration was calculated as the standard deviation between the rates at different protein concentrations. A $t$ test was performed to verify whether differences between the association rates of the $\mathrm{Cc}: \mathrm{CcP}$ complexes are statistically significant. The Bonferroni correction with $n=5$ was applied when comparing the data of a CcP variant with the other five variants (Tables S5 and S6). The analysis of the data suggests that dismutation of CpdII (reaction 4) is much slower than the other reactions. Nonetheless, the occurrence of this reaction explains the increase in the absorbance at $416 \mathrm{~nm}$ visible in kinetic traces obtained at low ionic strengths.

\section{RESULTS}

Rearranging the CcP Charge Distribution Creates New Interaction Sites. Electrostatic interactions play an important role in the complex of $\mathrm{Cc}$ and $\mathrm{CcP}$ and appear to guide $\mathrm{Cc}$ to the binding site for fast electron transfer. ${ }^{45-48}$ To test the degree to which the electrostatic interactions are optimized for the association between $\mathrm{Cc}$ and $\mathrm{CcP}$, we produced five $\mathrm{CcP}$ variants with a different charge distribution on their surface (Figure 1a,b). The CcP mutants were designed with three strategies, creation of an additional negative patch, neutralization of the negative charges in the wild type binding site for $\mathrm{Cc}$, or a combination of both. Wild type $\mathrm{CcP}$ is named here CcP_A. In variants CcP_B and CcP_D, an additional negative patch was introduced (change in charge of -8 ) on the side and the back of $\mathrm{CcP}$, respectively. Variants $\mathrm{CcP}$ C $\mathrm{C}$ and CCP_E carry the same additional charges as CCP_B and CcP_D and have $a+8$ charge change around the stereospecific binding site as defined by the structures of the wild type complex determined by crystallography and nuclear magnetic resonance (NMR), ${ }^{16,29}$ resulting in a net charge change of zero. Finally, CcP_F has the mutations only in the stereospecific binding site, resulting a charge change of +8 and rendering $\mathrm{CcP}$ close to neutral. Some data on CcP_A and
CcP_B were reported in a previous paper but are included here for the sake of completeness. ${ }^{27}$

Monte Carlo simulations in which $\mathrm{Cc}$ was docked to $\mathrm{CcP}$ a large number of times based solely on electrostatic interactions and steric constraints showed that the negative charges introduced on the surface of $\mathrm{CcP}$ attract $\mathrm{Cc}$ to the new sites (Figure 1c). The new patches strongly attract $\mathrm{Cc}$, in both CcP_B and CcP_D, and this effect is further enhanced in $\mathrm{CcP}_{-}^{-} \mathrm{C}$ and $\mathrm{CcP}_{-}^{-} \mathrm{E}$, suggesting that in all four mutants the encounter states would have Cc mostly or entirely at the new patches. Note that in the stereospecific complex next to electrostatic interactions, other favorable interactions are present in the WT type complex that are not modeled in these calculations, so it is expected that the shift in the interactions for the entire complex could be smaller than that shown in Figure 1c. For CcP_F, the calculations show a more even distribution of $\mathrm{Cc}$ around $\mathrm{CcP}$, as expected for a nearly neutral partner.

The energy distribution of the encounter complexes formed by the WT complex (Figure 1d) ranges from 0 to approximately $-10 \mathrm{kcal} / \mathrm{mol}$. The energy of most populated encounters is between approximately -4 and $-7 \mathrm{kcal} / \mathrm{mol}$; nevertheless, a significant fraction of the encounters, $\sim 25 \%$, has an energy between -3 and $1 \mathrm{kcal} / \mathrm{mol}$. The encounter complexes formed by $\mathrm{Cc}$ in the presence of $\mathrm{CcP} \_\mathrm{B}-\mathrm{CcP} \mathrm{E}$ show that the encounters are distributed in more narrow peaks shifted to more negative energy compared to the WT encounter complexes, suggesting that they have a more favorable interaction than in the $\mathrm{Cc}$ : $\mathrm{CcP} \_\mathrm{A}$ complex. In particular, the most favorable interactions are formed between $\mathrm{Cc}$ and $\mathrm{CcP} \_\mathrm{B}$, with a peak at $-10 \mathrm{kcal} / \mathrm{mol}$. As expected, the least favorable encounters are formed by the Cc:CcP_F complex, which presents a very narrow peak centered on $-\overline{0.5}$ $\mathrm{kcal} / \mathrm{mol}$.

CcP Turnover Involves Two Reduction Steps. Cc and $\mathrm{CcP}$ have high association $\left(k_{\mathrm{a}}\right)$ and dissociation $\left(k_{-\mathrm{a}}\right)$ rate constants: $k_{\mathrm{a}}=10^{8}-10^{9} \mathrm{M}^{-1} \mathrm{~s}^{-1}$ at an ionic strength of 200 $\mathrm{mM}^{39}$ and $k_{-\mathrm{a}} \geq 6400 \mathrm{~s}^{-1}$. $^{27}$ The encounter complex represents $30 \%$ of the total complex; ${ }^{29,47}$ the affinity is in the micromolar range $\left(K_{\mathrm{D}}=5 \mu \mathrm{M}\right),{ }^{49}$ and $\mathrm{ET}$ is fast $(>50000$ $\left.\mathrm{s}^{-1}\right) .{ }^{50}$ To establish the effect of charge redistribution on the formation of the stereospecific, electron transfer active complex, the rate of oxidation of reduced $\mathrm{Cc}, \mathrm{Cc}\left(\mathrm{Fe}^{2+}\right)$, was measured for the different $\mathrm{CcP}$ variants. Stopped flow experiments were performed following the work by Miller et al. ${ }^{39} \mathrm{Cc}\left(\mathrm{Fe}^{2+}\right)$ oxidation was followed by measuring the change in absorbance at $416 \mathrm{~nm}$ over time in the cycle of reactions that allows $\mathrm{CcP}$ to reduce $\mathrm{H}_{2} \mathrm{O}_{2}$ to water. First, resting state $\mathrm{CcP}, \mathrm{CcP}\left(\mathrm{Fe}^{3+}\right)$, is reacted with hydrogen peroxide to create compound I (CpdI, reaction I). ${ }^{51-54}$ Then, after mixing with $\mathrm{Cc}\left(\mathrm{Fe}^{2+}\right)$, a first molecule of $\mathrm{Cc}$ is oxidized to yield compound II (CpdII) and $\mathrm{Cc}\left(\mathrm{Fe}^{3+}\right)$ (reaction II). A second $\mathrm{Cc}\left(\mathrm{Fe}^{2+}\right)$ molecule can then reduce $\mathrm{CpdII}$ to resting state $\mathrm{CcP}$ (reaction III). ${ }^{55}$ Reactions II and III both contribute to the change in absorbance at $416 \mathrm{~nm}$. Furthermore, a slow regain of absorbance was observed, which we attributed to dismutation of CpdII into CpdI and resting state $\mathrm{CcP}$ (reaction IV).

$$
\begin{aligned}
& \mathrm{CcP}\left(\mathrm{Fe}^{3+}\right)+\mathrm{H}_{2} \mathrm{O}_{2} \rightarrow \mathrm{CpdI}+\mathrm{H}_{2} \mathrm{O} \\
& \mathrm{Cc}\left(\mathrm{Fe}^{2+}\right)+\mathrm{CpdI} \stackrel{k(\mathrm{II})}{\longrightarrow} \mathrm{Cc}\left(\mathrm{Fe}^{3+}\right)+\mathrm{CpdII}
\end{aligned}
$$


a)

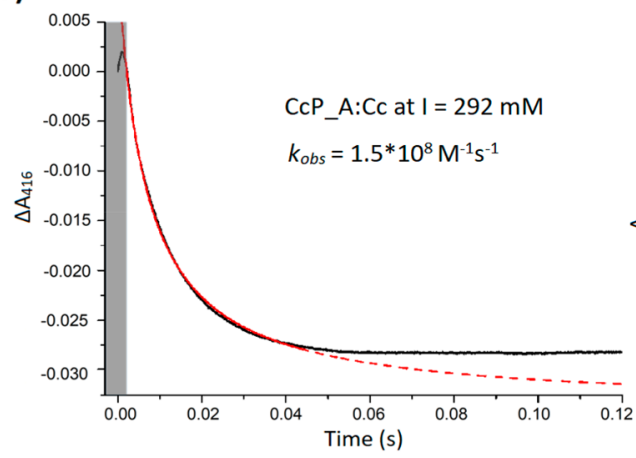

b)

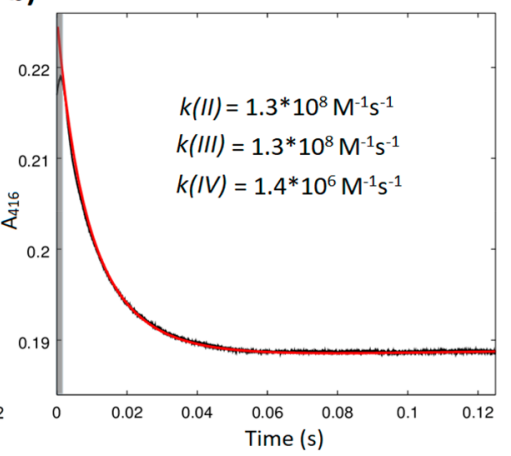

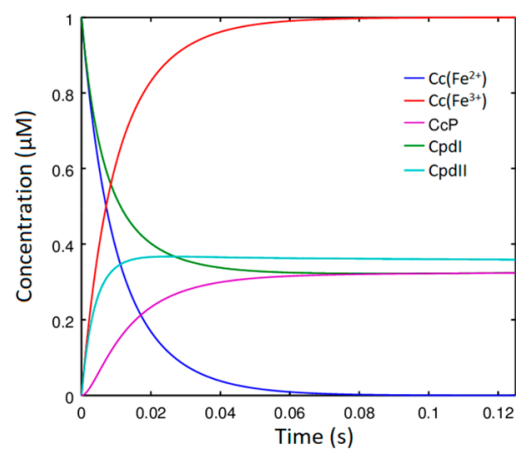

Figure 2. Comparison between fitting and simulation of the reaction performed by Cc and CcP_A at an ionic strength of $292 \mathrm{mM}$ measured at the stopped flow. A gray area covers the first part of the measured data affected by a stopped flow artifact and thus excluded from the analysis. (a) Fitting of the fast decay to eq 1 (see Materials and Methods). The data are shown as a solid black line, and the fit is represented by the red line. The extrapolation of the fit is shown as a dashed line. (b) Simulation (red line) of reactions II-IV to fit the same data (black) as in panel a (left). Concentrations over time of all of the species involved in the $\mathrm{Cc}$ : $\mathrm{CcP}$ cycle as derived from the simulation (right).

$$
\mathrm{Cc}\left(\mathrm{Fe}^{2+}\right)+\mathrm{CpdII} \stackrel{k(\mathrm{III})}{\longrightarrow} \mathrm{Cc}\left(\mathrm{Fe}^{3+}\right)+\mathrm{CcP}\left(\mathrm{Fe}^{3+}\right)+\mathrm{H}_{2} \mathrm{O}
$$

$$
2 \mathrm{CpdII} \stackrel{k(\mathrm{IV})}{\longrightarrow} \mathrm{CpdI}+\mathrm{CcP}\left(\mathrm{Fe}^{3+}\right)
$$

Immediately after mixing, reaction II dominates because the concentration of CpdI is maximal and that of CpdII still zero. Reaction II can be broken up into several steps (reaction IIa):

$$
\begin{aligned}
& \mathrm{Cc}\left(\mathrm{Fe}^{2+}\right)+\mathrm{C} \text { dI } \\
& \stackrel{k_{\text {a }}}{\rightleftarrows} \mathrm{Cc}\left(\mathrm{Fe}^{2+}\right): \text { CpdI } \\
& \stackrel{k_{-\mathrm{a}}}{\stackrel{k_{\text {et }}}{\rightarrow}} \mathrm{Cc}\left(\mathrm{Fe}^{3+}\right): \text { CpdII } \\
& \underset{k_{\mathrm{d}}}{\stackrel{k_{\text {-d }}}{\rightleftarrows}} \mathrm{Cc}\left(\mathrm{Fe}^{3+}\right)+\text { CpdII }
\end{aligned}
$$

The observed color change occurs after the electron transfer step $\left(k_{\text {et }}\right)$, and thus, the observed rate constant in the stopped flow trace, $k_{\mathrm{obs}}$, describes the first two parts of reaction IIa. It can readily be shown that under pseudo-steady state conditions $\left[\frac{\partial \mathrm{Cc}\left(\mathrm{Fe}^{2+}\right): \mathrm{CpdI}}{\partial t}=\frac{\partial \mathrm{Cc}\left(\mathrm{Fe}^{3+}\right): \mathrm{CpdII}}{\partial t}=0\right]$ eq 8 holds, giving the relation between the simulated rate $k$ (II) and the association rate constant $k_{\mathrm{a}}$ :

$$
k(\mathrm{II})=\frac{k_{\mathrm{a}} k_{\mathrm{et}}}{k_{-\mathrm{a}}+k_{\mathrm{et}}}
$$

To test whether the pseudo-steady state assumption is applicable, the $k_{\text {obs }}$ was obtained by fitting the initial part of the curve and the complete curve was simulated using differential equations that describe reactions II-IV, yielding estimates of $k(\mathrm{II}), k(\mathrm{III})$, and $k(\mathrm{IV})$. The two methods are illustrated in Figure 2 and Figure S1. Note that the fitting method using the analytical solution given in eq 1 can be applied only at equal concentrations of $\mathrm{Cc}$ and $\mathrm{CcP}$ and neglects reactions other than reaction II, so it fits only the initial part of the decay (Figure 2). The remaining part is strongly affected by the other reactions. The simulation method covers the entire curve and has the advantage of not being limited to a specific ratio of concentrations between the proteins, enabling us to evaluate the variation in the obtained rate due to concentration errors. This approach results in a more reliable error estimation for the rate constants. Excellent simulations could be obtained, but it should be noted that under some conditions there is correlation between the obtained rate constants; therefore, the three variables were not completely independent in all data sets (Table S3). Because of this correlation between parameters, we used simulations rather than fittings of the differential equations to avoid ending up in unrealistic local minima or with extreme values. For the analysis of the mutants, the primary interest is in $k$ (II). The values for this rate constant obtained by simulation matched the fitted $k_{\text {obs }}$ values (Table S4), indicating that eq 8 is approximated well and $k$ (II) can be used as a lower limit for association rate constant $k_{\mathrm{a}}$. For WT Cc and CcP, the electron transfer rate $\left(k_{\mathrm{et}}\right)$ is much larger than the dissociation rate constant $\left(k_{-\mathrm{a}}\right)$, so the observed rate constant approximates the association rate constant, $k(\mathrm{II}) \approx k_{\mathrm{a}}{ }^{12,56}$ In the remainder of the work, simulations were used for data analysis.

Cc Association Rates Vary Strongly for CcP Variants. Due to the favorable electrostatic interactions between $\mathrm{Cc}$ and $\mathrm{CcP}$, the association rate constant for the WT proteins is high at low ionic strengths and strongly decreases with an increasing salt concentration (Figure 3). ${ }^{18,20-23}$ It is estimated that at high salt concentrations, at an ionic strength of $1.25 \mathrm{M}$, all of the mutants converge to the same $k_{\mathrm{a}}$. At a low salt concentration, the complexes formed by $\mathrm{Cc}$ and $\mathrm{CcP}$ variants $\mathrm{A}, \mathrm{B}$, and $\mathrm{D}$ have a high $k_{\mathrm{a}}$, likely to be very close to the diffusion limit (Figure 3). At ionic strength values between 292 and $622 \mathrm{mM}$ (range of $17-25 \mathrm{mM}^{1 / 2}$ on the horizontal scale in Figure 3), the association rate constant for binding of $\mathrm{Cc}$ to $\mathrm{CcP}$ variant $\mathrm{B}$ is slightly higher than with CcP_A (Table S5). Similarly, CcP_D is slightly faster than CcP_A in the ionic strength range of 392-622 mM (Figure 3 and Table S5). Only at ionic strength values of $<122 \mathrm{mM}$, the association with CcP_D is slightly slower than with wild type CcP. The association rate constant for binding of $\mathrm{Cc}$ to $\mathrm{CcP}$ variants $\mathrm{C}$, $\mathrm{E}$, and $\mathrm{F}$ is strongly reduced compared to that for CcP_A. At ionic strength values of $>170 \mathrm{mM}\left(14 \mathrm{mM}^{1 / 2}\right)$, the complexes formed by $\mathrm{Cc}$ and the mutants of $\mathrm{CcP} C$ and E show the same basal association rate as the complex formed with CcP_F. Only a significant difference is present at an ionic strength of $44 \mathrm{mM}$ $\left(7 \mathrm{mM}^{1 / 2}\right)$ (Table S5). Under this condition, mutant CcP_C binds faster to $\mathrm{Cc}$ than $\mathrm{CcP}_{-} \mathrm{E}$ and CcP_F. 


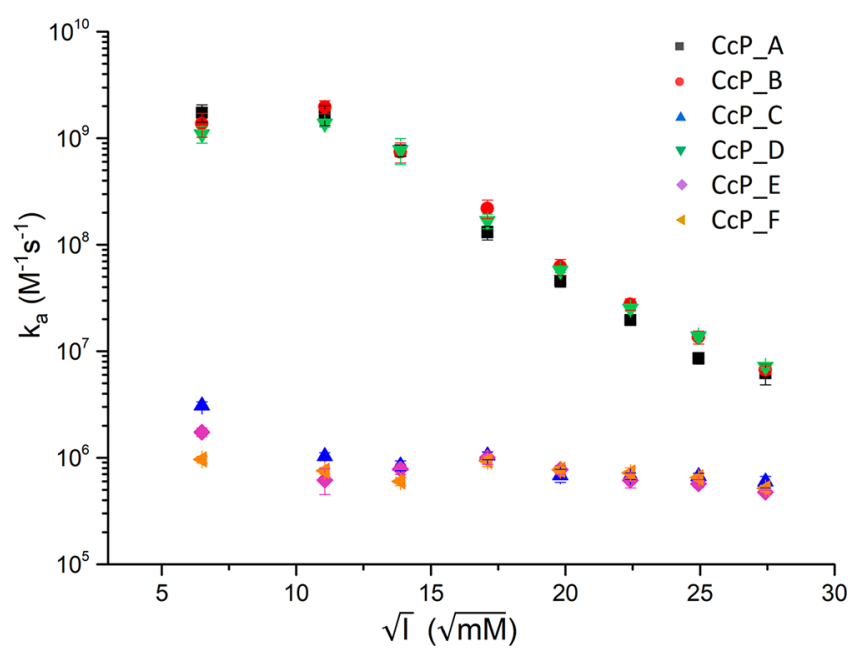

Figure 3. Rates of association $\left(k_{\mathrm{a}}\right)$ between $\mathrm{Cc}$ and the $\mathrm{CcP}$ variants. The $k_{\mathrm{a}}$ values, plotted as a function of the square root of the ionic strength, were obtained from the simulations of the stopped flow kinetics. Errors were calculated as the standard deviation between replicates and simulations performed at different $\mathrm{CcP}$ concentrations (see Materials and Methods for details).

\section{DISCUSSION}

To test the importance of the charge distribution on the surface of $\mathrm{CcP}$ for the interactions with $\mathrm{Cc}$, five $\mathrm{CcP}$ mutants were created with different electrostatic potential and charge distribution on their surfaces (Figure 1a,b). Monte Carlo simulations were performed for the wild type and mutated $\mathrm{Cc}$ :CcP complexes. The energy distributions for the $\mathrm{Cc}$ : CcP complexes (Figure 1d) show that the charge interactions between $\mathrm{Cc}$ and the $\mathrm{CcP}$ mutants, apart from the more neutral CcP_F, are more favorable than for the WT complex, in line with the increased overall negative charge on mutants $\mathrm{CcP} B$ and $\mathrm{CcP}$ D. Interestingly, also mutants $\mathrm{CcP} \mathrm{C}_{2}$ and $\mathrm{CcP}_{-} \mathrm{E}$, which have the same net charge as $\mathrm{CcP}_{-} \mathrm{A}$, yield somewhat more favorable interactions. The reason could be that in the mutants an artificially highly negative patch is constructed. Often, charges on protein surfaces are compensated by nearby residues to ensure better protein stability. The simulations also demonstrate that these new patches strongly attract $\mathrm{Cc}$, an effect that is enhanced further for the mutants in which the charges in the normal binding site are removed [ $\mathrm{CcP}_{-} \mathrm{C}$ and CcP_E (Figure 1c)]. Thus, it can be expected that the encounter complex of $\mathrm{Cc}$ and $\mathrm{CcP}$ is shifted toward binding at the new patches, which we demonstrated before for CcP B using paramagnetic NMR spectroscopy. ${ }^{27}$ In the Monte Carlo simulations, it is assumed that all residues in the negative patches are charged but the possibility that mutual repulsion leads to increased $\mathrm{p} K_{\mathrm{a}}$ values cannot be excluded, rendering the patches with a lower net charge at the experimental $\mathrm{pH}$ value of 6.0. Furthermore, the simulations consider only electrostatic interactions and therefore neglect other favorable interactions that could play an important role at the stereospecific binding site. To determine whether productive complex formation, leading to $\mathrm{ET}$ from $\mathrm{Cc}$ to $\mathrm{CpdI}$, can occur in the mutant complexes, stopped flow experiments were performed and association rate constants determined. The six $\mathrm{CcP}$ variants analyzed can be divided in two groups: $\mathrm{CcP}$ variants with the wild type charges in the binding site intact $(\mathrm{CcP} A \mathrm{~A}, \mathrm{CcP} B$, and $\left.\mathrm{CcP}_{-} \mathrm{D}\right)$ and variants in which these charges were neutralized $\left(\mathrm{CcP}_{-} \mathrm{C}, \mathrm{C} \mathrm{P} \_\mathrm{E}\right.$, and $\left.\mathrm{CcP}_{-} \mathrm{F}\right)$. The rate constants of association of the first group of $\mathrm{CcP}$ variants are similar and strongly dependent on the ionic strength due to the favorable electrostatic interactions between $\mathrm{Cc}$ and $\mathrm{CcP}$ (Figure $3)^{18,20-23}$ The second group instead presents a much lower association rate constant that is nearly independent of ionic strength. Clearly, the charges around the stereospecific binding site for $\mathrm{Cc}$ are therefore fundamental for the formation of a productive encounter complex and efficient ET, in agreement with previous literature. ${ }^{24-26}$ All variants of CcP bind the heme group, yielding ultraviolet-visible spectra similar to those of WT CcP, and they can all form CpdI and be reduced by Cc. Thus, the mutations do not affect the integrity of $\mathrm{CcP}$. We cannot exclude, however, the possibility that the mutations around the stereospecific binding site subtly affect the protein surface, thus contributing to a reduced level of formation of the ET active complex.

It can be expected that at a high salt concentration, at an ionic strength of $\sim 1.25 \mathrm{M}$, all variants converge to the same basal association rate constant of $\sim 5 \times 10^{5} \mathrm{M}^{-1} \mathrm{~s}^{-1}$, which is independent of ionic strength. The highest value observed $\left(k_{\mathrm{a}}\right.$ $=2 \times 10^{9} \mathrm{M}^{-1} \mathrm{~s}^{-1}$ ) shows that the electrostatic interactions can increase the rate 4000 -fold compared to that of ET by random collision. The obtained rate constant $k$ (II) is a lowerlimit estimate of $k_{\mathrm{a}}$ (eq 8 ), so the actual $k_{\mathrm{a}}$ could be even larger; however, it seems to approach the diffusion limit, which is suggested by the leveling off of the $k_{\mathrm{a}}$ at a very low ionic strength (Figure 3). Another explanation for such leveling off relates to the balance of monopole-dipole effects on the interactions as a function of ionic strength. ${ }^{57-59}$

The results indicate that the charges added on the surface of CcP_B and CcP_D do not negatively affect the formation of the stereospecific complex. We previously reported that the enlarged encounter region on $\mathrm{CcP}$ _B in fact slightly increases the association rate constant. ${ }^{27}$ Our hypothesis is that the added charges enhance the electrostatic interactions between $\mathrm{Cc}$ and $\mathrm{CcP}$, outweighing the negative effect of interacting at a site more distant from the stereospecific binding site. In other words, the additional charges enhance the probability of productive complex formation and binding at the new patch therefore, by definition, represents a productive encounter. Surprisingly, a similar result is observed with CcP_D. The association rate constants between $\mathrm{Cc}$ and $\mathrm{CcP} D \mathrm{D}^{-}$at ionic strength values between 392 and $622 \mathrm{mM}$ (from 20 to 25 $\mathrm{mM}^{1 / 2}$ ) are significantly higher than the association measured for the wild type complex (Table S5). The differences are small for both CcP_B and CcP_D but are consistently higher than for CcP_A in that ionic strength range (Tables S5 and S6). The rigid body Monte Carlo simulations show that $\mathrm{Cc}$ is strongly attracted by the new negative patch of $\mathrm{CcP} \_\mathrm{D}$, located on the opposite side of the protein with respect to the stereospecific binding site (Figure 1), and it still results in a slight increase in the rate of productive complex formation at moderate to high ionic strength values. Thus, analogous to $\mathrm{CcP}$ B , we conclude that the positive effect of the extra charges outweighs the remote binding relative to the reaction site, yielding more productive encounters for this variant. At ionic strength values of $<122 \mathrm{mM}\left(11 \mathrm{mM}^{1 / 2}\right)$, the association rate constants between $\mathrm{Cc}$ and $\mathrm{CcP}$ D are significantly lower than for the WT complex. This suggests that at low salt concentrations the interaction between $\mathrm{Cc}$ and the new negative patch on $\mathrm{CcP} \_\mathrm{D}$ reduces the probability of $\mathrm{Cc}$ finding the stereospecific binding site. Similar biphasic behavior with 
reduced ET rates at low ionic strengths was reported before, for example, for plastocyanin and plant cytochrome $f .^{60}$

Perhaps the most surprising result is that the new patches cannot compensate at all for the loss of the charge interactions at the stereospecific binding site. At ionic strength values of $>192 \mathrm{mM}\left(14 \mathrm{mM}^{1 / 2}\right)$, the complexes formed by $\mathrm{Cc}$ and CcP_C and CcP_E, which have a net charge identical to that of $\overline{\mathrm{WT}} \mathrm{CcP}$, show the same, basal association rate constant as the complex formed with CcP_F, which has a charge change of +8 relative to WT $\mathrm{CcP}$ and is overall close to neutral. Therefore, whereas the added charged patches appear to enhance the productive encounter formation in the presence of the charges in the stereospecific binding site, they have no effect on productive complex formation whatsoever if the binding site charges have been neutralized, at least at moderate and high ionic strengths. An effect is observed only at an ionic strength of $44 \mathrm{mM}\left(7 \mathrm{mM}^{1 / 2}\right)$, under which condition the association rate constants decrease in the following order: CcP_C > CcP_E > CcP_F. Apparently, at this salt concentration, the electrostatic interactions are strong enough to result in slightly more productive encounters, with the largest effect for the charged patch that is closest to the stereospecific binding site $\left(\mathrm{CcP}_{-} \mathrm{C}\right)$. Similar results were obtained by Harel et al., ${ }^{10}$ who studied the complex formed by TEM1 and BLIP. Creating different mutants of BLIP and using stopped flow association rate measurements in combination with Brownian dynamics simulations, they showed that increasing the number of charge interactions that led to successful binding (fruitful trajectories) increases the rate of association between the two proteins.

\section{CONCLUSIONS}

Our results indicate that in the presence of favorable charges in the binding site, the efficiency of active protein complex formation depends more on the overall strength of the electrostatic interactions than on the distribution of charges on the protein surface. Nonetheless, the position of the charges on the protein surface can slightly affect productive complex formation, depending on the ionic strength conditions. At moderate and high ionic strengths, additional charges enhance it, whereas at low ionic strengths, it can be reduced, as seen for CcP D. The neutralization of the charges at the stereospecific binding site for Cc strongly affects the association of the two proteins, abolishing the electrostatic interactions entirely, as judged from the ionic strength independence of complex formation with CcP_F. Addition of highly charged patches distant from the stereospecific binding site only slightly enhances complex formation at low ionic strengths and as no effect at moderate or high ionic strengths. In this case, although the Monte Carlo simulations clearly show that electrostatic interactions are strong for $\mathrm{CcP}_{-} \mathrm{C}$ and $\mathrm{CcP} E$, apparently no trajectories are present for $\mathrm{Cc}$ to diffuse from the charged patch toward the stereospecific binding site, rather than to dissociate from $\mathrm{CcP} .{ }^{10,14}$ Therefore, in the absence of the negative charges in the stereospecific binding site, the encounters at the new patches have become futile ones.

\section{ASSOCIATED CONTENT}

\section{s) Supporting Information}

The Supporting Information is available free of charge at https://pubs.acs.org/doi/10.1021/acs.biochem.1c00021.
Example of kinetics at low ionic strengths, list of mutations in the $\mathrm{CcP}$ variants, extinction coefficients of the proteins, association rate constants, and script of the kinetic simulations (PDF)

\section{Accession Codes}

CYC1, P00044; CCP1, P00431.

\section{AUTHOR INFORMATION}

\section{Corresponding Author}

Marcellus Ubbink - Leiden University, Institute of Chemistry, Einsteinweg 55, 2333 CC Leiden, The Netherlands; 이이.org/0000-0002-2615-6914; Email: m.ubbink@ chem.leidenuniv.nl

\section{Authors}

Antonella Di Savino - Leiden University, Institute of Chemistry, Einsteinweg 55, 2333 CC Leiden, The Netherlands

Johannes M. Foerster - University of Bayreuth, Computational Biochemistry, Universitätsstraße 30, NW I, 95447 Bayreuth, Germany; ○ orcid.org/0000-0003-04429413

G. Matthias Ullmann - University of Bayreuth, Computational Biochemistry, Universitätsstraße 30, NW I, 95447 Bayreuth, Germany; 이이이.org/0000-0002-6350$798 \mathrm{X}$

Complete contact information is available at: https://pubs.acs.org/10.1021/acs.biochem.1c00021

\section{Author Contributions}

A.D.S., J.M.F., and M.U. performed research and analyzed data. A.D.S., J.M.F., G.M.U., and M.U. wrote the paper.

\section{Funding}

This work was supported by The Netherlands Organisation for Scientific Research (NWO-CW Grant 711.013.007 to M.U.), by the Deutsche Forschungsgemeinschaft (DFG Grant SFB 1357, TP C03 to G.M.U.), and the study program "Biological Physics" of the Elite Network of Bavaria (J.M.F. and G.M.U.).

Notes

The authors declare no competing financial interest.

\section{ACKNOWLEDGMENTS}

The authors acknowledge Dr. Valentina Masarotto for assistance with the statistical analysis.

\section{REFERENCES}

(1) McLendon, G. (1991) Control of biological electron transport via molecular recognition and binding: The "velcro" model. In LongRange Electron Transfer in Biology, p 159, Springer, Berlin.

(2) Kim, Y. C., Tang, C., Clore, G. M., and Hummer, G. (2008) Replica exchange simulations of transient encounter complexes in protein-protein association. Proc. Natl. Acad. Sci. U. S. A. 105, 1285512860.

(3) Van de Water, K., van Nuland, N. A. J., and Volkov, A. N. (2014) Transient protein encounters characterized by paramagnetic NMR. Chem. Sci. 5, 4227-4236.

(4) Scanu, S., Foerster, J. M., Ullmann, G. M., and Ubbink, M. (2013) Role of hydrophobic interactions in the encounter complex formation of the plastocyanin and cytochrome $f$ complex revealed by paramagnetic NMR spectroscopy. J. Am. Chem. Soc. 135, 7681-7692.

(5) Sugase, K., Dyson, H. J., and Wright, P. E. (2007) Mechanism of coupled folding and binding of an intrinsically disordered protein. Nature 447, 1021-U11. 
(6) Camacho, C. J., Weng, Z., Vajda, S., and DeLisi, C. (1999) Free energy landscapes of encounter complexes in protein-protein association. Biophys. J. 76, 1166-1178.

(7) Camacho, C. J., Kimura, S. R., DeLisi, C., and Vajda, S. (2000) Kinetics of desolvation-mediated protein-protein binding. Biophys. J. 78, 1094-1105.

(8) Camacho, C. J., and Vajda, S. (2002) Protein-protein association kinetics and protein docking. Curr. Opin. Struct. Biol. 12, 36-40.

(9) Rajamani, D., Thiel, S., Vajda, S., and Camacho, C. J. (2004) Anchor residues in protein-protein interactions. Proc. Natl. Acad. Sci. U. S. A. 101, 11287-11292.

(10) Harel, M., Spaar, A., and Schreiber, G. (2009) Fruitful and futile encounters along the association reaction between proteins. Biophys. J. 96, 4237-4248.

(11) Fawzi, N. L., Doucleff, M., Suh, J. Y., and Clore, G. M. (2010) Mechanistic details of a protein-protein association pathway revealed by paramagnetic relaxation enhancement titration measurements. Proc. Natl. Acad. Sci. U. S. A. 107, 1379-1384.

(12) Schreiber, G., Haran, G., and Zhou, H. X. (2009) Fundamental aspects of protein-protein association kinetics. Chem. Rev. 109, 839860.

(13) An, S. Y., Kim, E.-H., and Suh, J.-Y. (2018) Facilitated protein association via engineered target search pathways visualized by paramagnetic NMR spectroscopy. Structure 26, 887-893.e2.

(14) Andrałojć, W., Hiruma, Y., Liu, W. M., Ravera, E., Nojiri, M., Parigi, G., Luchinat, C., and Ubbink, M. (2017) Identification of productive and futile encounters in an electron transfer protein complex. Proc. Natl. Acad. Sci. U. S. A. 114, E1840-E1847.

(15) Strickland, M., Kale, S., Strub, M. P., Schwieters, C. D., Liu, J., Peterkofsky, A., and Tjandra, N. (2019) Potential regulatory role of competitive encounter complexes in paralogous phosphotransferase systems. J. Mol. Biol. 431, 2331-2342.

(16) Pelletier, H., and Kraut, J. (1992) Crystal structure of a complex between electron transfer partners, cytochrome $c$ peroxidase and cytochrome c. Science (Washington, DC, U. S.) 258, 1748-1755.

(17) Stemp, E. D. A., and Hoffman, B. M. (1993) Cytochrome $c$ peroxidase binds two molecules of cytochrome $c$ : evidence for a lowaffinity, electron-transfer-active site on cytochrome $c$ peroxidase. Biochemistry 32, 10848-10865.

(18) Zhou, J. S., and Hoffman, B. M. (1994) Stern-Volmer in reverse - 2/1 stoichiometry of the cytochrome $c$ cytochrome $c$ peroxidase electron-transfer complex. Science (Washington, DC, U. S.) 265, 16931696.

(19) Mauk, M. R., Ferrer, J. C., and Mauk, A. G. (1994) Proton linkage in formation of the cytochrome $c$-cytochrome $c$ peroxidase complex: Electrostatic properties of the high- and low-affinity cytochrome binding sites on the peroxidase. Biochemistry 33, 12609-12614.

(20) Van de Water, K., Sterckx, Y. G. J., and Volkov, A. N. (2015) The low-affinity complex of cytochrome $c$ and its peroxidase. Nat. Commun. 6, 7073.

(21) Matthis, A. L., and Erman, J. E. (1995) Cytochrome c peroxidase-catalyzed oxidation of yeast iso-1 ferrocytochrome $c$ by hydrogen peroxide. Ionic strength dependence of the steady-state parameters. Biochemistry 34, 9985-9990.

(22) Matthis, A. L., Vitello, L. B., and Erman, J. E. (1995) Oxidation of yeast iso- 1 ferrocytochrome $c$ by yeast cytochrome $c$ peroxidase compounds I and II. Dependence upon ionic strength. Biochemistry 34, 9991-9999.

(23) McLendon, G., Zhang, Q., Billstone, V., Wallin, S. A., Miller, R. M., Spears, K. G., and Hoffman, B. M. (1993) Thermodynamic and kinetic aspects of binding and recognition in the cytochrome $\mathrm{c} /$ cytochrome c peroxidase complex. J. Am. Chem. Soc. 115, 3665-3669.

(24) Erman, J. E., Vitello, L. B., Pearl, N. M., Jacobson, T., Francis, M., Alberts, E., Kou, A., and Bujarska, K. (2015) Binding of yeast cytochrome $c$ to forty-four charge-reversal mutants of yeast cytochrome $c$ peroxidase: isothermal titration calorimetry. Biochemistry $54,4845-4854$.
(25) Pearl, N. M., Jacobson, T., Arisa, M., Vitello, L. B., and Erman, J. E. (2007) Effect of single-site charge-reversal mutations on the catalytic properties of yeast cytochrome $c$ peroxidase: Mutations near the high-affinity cytochrome $c$ binding site. Biochemistry 46, 82638272.

(26) Pearl, N. M., Jacobson, T., Meyen, C., Clementz, A. G., Ok, E. Y., Choi, E., Wilson, K., Vitello, L. B., and Erman, J. E. (2008) Effect of single-site charge-reversal mutations on the catalytic properties of yeast cytochrome $c$ peroxidase: Evidence for a single, catalytically active, cytochrome $c$ binding domain. Biochemistry 47, 2766-2775.

(27) Di Savino, A., Foerster, J., La Haye, T., Blok, A., Timmer, M., Ullmann, M., and Ubbink, M. (2020) Efficient encounter complex formation and electron transfer to cytochrome $c$ peroxidase with an additional, distant electrostatic binding site. Angew. Chem., Int. Ed. 59, 23239-23243.

(28) Goodin, D. B., Davidson, M. G., Roe, J. A., Mauk, A. G., and Smith, M. (1991) Amino-acid substitutions at tryptophan-51 of cytochrome $c$ peroxidase - Effects on coordination, species preference for cytochrome $c$, and electron-transfer. Biochemistry 30, 4953-4962.

(29) Volkov, A. N., Worrall, J. A. R., Holtzmann, E., and Ubbink, M. (2006) Solution structure and dynamics of the complex between cytochrome $c$ and cytochrome $c$ peroxidase determined by paramagnetic NMR. Proc. Natl. Acad. Sci. U. S. A. 103, 18945-18950.

(30) Schilder, J., Lohr, F., Schwalbe, H., and Ubbink, M. (2014) The cytochrome $c$ peroxidase and cytochrome $c$ encounter complex: The other side of the story. FEBS Lett. 588, 1873-1878.

(31) Teske, J. G., Savenkova, M. I., Mauro, J. M., Erman, J. E., and Satterlee, J. D. (2000) Yeast cytochrome $c$ peroxidase expression in Escherichia coli and rapid isolation of various highly pure holoenzymes. Protein Expression Purif. 19, 139-147.

(32) Pollock, W. B., Rosell, F. I., Twitchett, M. B., Dumont, M. E., and Mauk, A. G. (1998) Bacterial expression of a mitochondrial cytochrome $c$. Trimethylation of lys 72 in yeast iso-1-cytochrome $c$ and the alkaline conformational transition. Biochemistry 37, 6124-6131.

(33) Brünger, A. T., and Karplus, M. (1988) Polar hydrogen positions in proteins: Empirical energy placement and neutron diffraction comparison. Proteins: Struct., Funct., Genet. 4, 148-156.

(34) Brooks, B. R., Bruccoleri, R. E., Olafson, B. D., States, D. J., Swaminathan, S., and Karplus, M. (1983) CHARMM: A program for macromolecular energy, minimization, and dynamics calculations. $J$. Comput. Chem. 4, 187-217.

(35) MacKerell, A. D., Bashford, D., Bellott, M., Dunbrack, R. L., Evanseck, J. D., Field, M. J., Fischer, S., Gao, J., Guo, H., Ha, S., Joseph-McCarthy, D., Kuchnir, L., Kuczera, K., Lau, F. T. K., Mattos, C., Michnick, S., Ngo, T., Nguyen, D. T., Prodhom, B., Reiher, W. E., Roux, B., Schlenkrich, M., Smith, J. C., Stote, R., Straub, J., Watanabe, M., Wiórkiewicz-Kuczera, J., Yin, D., and Karplus, M. (1998) All-atom empirical potential for molecular modeling and dynamics studies of proteins. J. Phys. Chem. B 102, 3586-3616.

(36) The PyMOL molecular graphics system, ver. 1.3, Schrödinger, LLC.

(37) Jurrus, E., Engel, D., Star, K., Monson, K., Brandi, J., Felberg, L. E., Brookes, D. H., Wilson, L., Chen, J., Liles, K., Chun, M., Li, P., Gohara, D. W., Dolinsky, T., Konecny, R., Koes, D. R., Nielsen, J. E., Head-Gordon, T., Geng, W., Krasny, R., Wei, G. W., Holst, M. J., McCammon, J. A., and Baker, N. A. (2018) Improvements to the APBS biomolecular solvation software suite. Protein Sci. 27, 112-128.

(38) Foerster, J. M., Poehner, I., and Ullmann, G. M. (2018) MCMap - A computational tool for mapping energy landscapes of transient protein-protein interactions. ACS Omega 3, 6465-6475.

(39) Miller, M. A., Liu, R. Q., Hahm, S., Geren, L., Hibdon, S., Kraut, J., Durham, B., and Millett, F. (1994) Interaction domain for the reaction of cytochrome $c$ with the radical and the oxyferryl heme in cytochrome $c$ peroxidase compound I. Biochemistry 33, 8686-8693.

(40) Hahm, S., Miller, M. A., Geren, L., Kraut, J., Durham, B., and Millett, F. (1994) Reaction of horse cytochrome $c$ with the radical and the oxyferryl heme in cytochrome $c$ peroxidase compound-I. Biochemistry 33, 1473-1480. 
(41) Coulson, A. F., Erman, J. E., and Yonetani, T. (1971) Studies on cytochrome $c$ peroxidase. XVII. Stoichiometry and mechanism of the reaction of compound ES with donors. J. Biol. Chem. 246, 917924.

(42) Ho, P. S., Hoffman, B. M., Kang, C. H., and Margoliash, E. (1983) Control of the transfer of oxidizing equivalents between heme iron and free radical site in yeast cytochrome $c$ peroxidase. J. Biol. Chem. 258, 4356-4363.

(43) Eaton, J. W., Bateman, D., Hauberg, S., and Wehbring, R. (2020) GNU Octave version 5.2 .0 manual: a high-level interactive language for numerical computations. https://www.gnu.org/software/ octave/doc/v5.2.0/.

(44) Margoliash, E., and Frohwirt, N. (1959) Spectrum of horseheart cytochrome $c$. Biochem. J. 71, 570-578.

(45) Gabdoulline, R. R., and Wade, R. C. (2001) Protein-protein association: investigation of factors influencing association rates by Brownian dynamics simulations. J. Mol. Biol. 306, 1139-1155.

(46) Northrup, S. H., Boles, J. O., and Reynolds, J. C. (1988) Brownian dynamics of cytochrome $c$ and cytochrome $c$ peroxidase association. Science (Washington, DC, U. S.) 241, 67-70.

(47) Bashir, Q., Volkov, A. N., Ullmann, G. M., and Ubbink, M. (2010) Visualization of the encounter ensemble of the transient electron transfer complex of cytochrome $c$ and cytochrome $c$ peroxidase. J. Am. Chem. Soc. 132, 241-247.

(48) Castro, G., Boswell, C. A., and Northrup, S. H. (1998) Dynamics of protein-protein docking: Cytochrome $c$ and cytochrome c peroxidase revisited. J. Biomol. Struct. Dyn. 16, 413-424.

(49) Worrall, J. A. R., Kolczak, U., Canters, G. W., and Ubbink, M. (2001) Interaction of yeast iso-1-cytochrome $c$ with cytochrome $c$ peroxidase investigated by $\mathrm{N}-15, \mathrm{H}-1$ heteronuclear NMR spectroscopy. Biochemistry 40, 7069-7076.

(50) Geren, L., Hahm, S., Durham, B., and Millett, F. (1991) Photoinduced electron-transfer between cytochrome $c$ peroxidase and yeast cytochrome $c$ labeled at cys-102 with(4-bromomethyl-4'methylbipyridine) bis(bypyridine) ruthenium2+. Biochemistry 30, 9450-9457.

(51) Mauro, J. M., Fishel, L. A., Hazzard, J. T., Meyer, T. E., Tollin, G., Cusanovich, M. A., and Kraut, J. (1988) Tryptophan-191 $\rightarrow$ Phenylalanine, a proximal-side mutation in yeast cytochrome $c$ peroxidase that strongly affects the kinetics of ferrocytochrome $c$ oxidation. Biochemistry 27, 6243-6256.

(52) Sivaraja, M., Goodin, D. B., Smith, M., and Hoffman, B. M. (1989) Identification by ENDOR of Trp191 as the free-radical site in cytochrome $c$ peroxidase compound ES. Science (Washington, DC, $U$. S.) $245,738-740$.

(53) Erman, J. E., Vitello, L. B., Mauro, J. M., and Kraut, J. (1989) Detection of an oxyferryl porphyrin $\pi$-cation-radical intermediate in the reaction between hydrogen peroxide and a mutant yeast cytochrome $c$ peroxidase. Evidence for tryptophan-191 involvement in the radical site of compound. Biochemistry 28, 7992-7995.

(54) Fishel, L. A., Farnum, M. F., Miller, M. A., Kraut, J., Mauro, J. M., Liu, Y., Tan, X. L., and Scholes, C. P. (1991) Compound I radical in site-directed mutants of cytochrome $c$ peroxidase as probed by electron paramagnetic resonance and electron-nuclear double resonance. Biochemistry 30, 1986-1996.

(55) Kim, K. L., Kang, D. S., Vitello, L. B., and Erman, J. E. (1990) Cytochrome $c$ peroxidase catalyzed oxidation of ferrocytochrome $c$ by hydrogen-peroxide - Ionic-strenght dependence of the steady-state rate parameters. Biochemistry 29, 9150-9159.

(56) Ho, P. S., Hoffman, B. M., Solomon, N., Kang, C. H., and Margoliash, E. (1984) Kinetics and energetics of intramolecular electron-transfer in yeast cytochrome $c$ peroxidase. Biochemistry 23, $4122-4128$.

(57) Koppenol, W. H., and Margoliash, E. (1982) The asymmetric distribution of charges on the surface of horse cytochrome c-Functional implications. J. Biol. Chem. 257, 4426-4437.

(58) Van Leeuwen, J. W. (1983) The ionic strength dependence of the rate of a reaction between two large proteins with a dipole moment. Biochim. Biophys. Acta, Protein Struct. Mol. Enzymol. 743, $408-421$

(59) Watkins, J. A., Cusanovich, M. A., Meyer, T. E., and Tollin, G. (1994) A "parallel plate" electrostatic model for bimolecular rate constants applied to electron transfer proteins. Protein Sci. 3, 21042114.

(60) Meyer, T. E., Zhao, Z. G., Cusanovich, M. A., and Tollin, G. (1993) Transient kinetics of electron transfer from a variety of $c$-type cytochromes to plastocyanin. Biochemistry 32, 4552-4559. 